Documentation et bibliothèques

DOCUMENTATION BIBLIOTHEQUES

\title{
Le programme de bibliotechnique au Collège Algonquin
}

\section{Jacques LeBlanc}

Volume 19, numéro 2, juin 1973

\section{Bibliotechnique}

URI : https://id.erudit.org/iderudit/1055810ar

DOI : https://doi.org/10.7202/1055810ar

Aller au sommaire du numéro

\section{Éditeur(s)}

Association pour l'avancement des sciences et des techniques de la documentation (ASTED)

\section{ISSN}

0315-2340 (imprimé)

2291-8949 (numérique)

Découvrir la revue

\section{Citer cet article}

LeBlanc, J. (1973). Le programme de bibliotechnique au Collège Algonquin. Documentation et bibliothèques, 19(2), 57-60. https://doi.org/10.7202/1055810ar

\section{Résumé de l'article}

La tendance de plus en plus accusée, chez les bibliotechniciens, à affirmer leur identité - illustrée par exemple lors de débats au dernier Congrès de l'ACBLF de même qu'un léger malaise prévalant dans certains milieux scolaires au sujet de leurs rapports de travail avec, d'une part, les bibliothécaires, de l'autre, les commis, représentent des " signes des temps » qu'aucun membre de notre profession ne peut se permettre d'ignorer. C'est dans cette perspective - élargir les horizons, faire pénétrer au coeur d'un cadre pour plusieurs largement périphérique - que le présent article se veut conçu, en présentant le plus objectivement possible ce qui existe, dans l'Ontario francophone, relativement au secteur des études de la bibliotechnique. Précisons que l'attention exclusive consacrée ici au Collège Algonquin relève du simple fait qu'il constitue la seule institution ontarienne à dispenser, dans ce domaine, un enseignement en français.
Tous droits réservés (C) Association pour l'avancement des sciences et des techniques de la documentation (ASTED), 1973
Ce document est protégé par la loi sur le droit d'auteur. L'utilisation des services d'Érudit (y compris la reproduction) est assujettie à sa politique d'utilisation que vous pouvez consulter en ligne.

https://apropos.erudit.org/fr/usagers/politique-dutilisation/ 


\section{Le programme de bibliotechnique au Collège Algonquin}

\author{
Jacques LeBlanc
}

Université d'Ottawa

La tendance de plus en plus accusée, chez les bibliotechniciens, à affirmer leur identité - illustrée par exemple lors de débats au dernier Congrès de l'ACBLF - de même qu'un léger malaise prévalant dans certains milieux scolaires au sujet de leurs rapports de travail avec, d'une part, les bibliothécaires, de l'autre, les commis, représentent des "signes des temps" qu'aucun membre de notre profession ne peut se permettre d'ignorer. C'est dans cette perspective - élargir les horizons, faire pénétrer au coeur d'un cadre pour plusieurs largement périphérique - que le présent article se veut conçu, en présentant le plus objectivement possible ce qui existe, dans l'Ontario francophone, relativement au secteur des études de la bibliotechnique. Précisons que l'attention exclusive consacrée ici au Collège Algonquin relève du simple fait qu'il constitue la seule institution ontarienne à dispenser, dans ce domaine, un enseignement en français.

Le Collège Algonquin des arts appliqués et de la technologie dessert la partie ontarienne de la vallée de l'Outaouais (soit les comtés de Renfrew, Lanark, Carleton, Prescott et Russell). Comme son nom l'indique, et à l'instar des établissements similaires en Ontario, il s'agit d'un collège communautaire - à peu près l'équivalent, sur le plan des études et des structures, du Cegep québécois; il sert la clientèle du post-secondaire dans le champ de la technologie, des arts appliqués et de l'enseignement professionnel. Ses programmes, tant pour les étudiants à temps plein que ceux à temps partiel, recouvrent une grande diversité de matières - de l'anglais et du journalisme à la climatisation et à la physique - et sont de durée variable (d'une à trois années selon les disciplines). Ils rejoignent, par l'intermédiaire de services d'éducation permanente et de centres de recyclage, un grand nombre de personnes déjà engagées dans le monde du travail. Par ses activités globales, le Collège qui, depuis 1969, a adopté une ambitieuse politique de bilinguisme, demeure à l'heure actuelle le seul en Ontario donnant, dans la mesure du possible, un enseignement dans les deux langues officielles. II s'avère, par le fait même, en mesure de répondre à la fois aux aspirations culturelles des francophones et aux besoins particuliers de la région en ce qui regarde le personnel bilingue.

C'est dans ce cadre général, et plus précisément à l'intérieur de la Faculté des arts appliqués, département d'études des services communautaires, que s'inscrit le cours de bibliotechnique. Le calendrier, réparti sur deux ans (4 semestres, 130 crédits, soit une moyenne de 25 heures/semaine), avec diplôme en français et en anglais - ce qui implique en fait deux coordonnateurs et deux programmes identiques en tous points - vise primordialement à préparer le futur bibliotechnicien à accomplir des tâches spécifiques sous la direction plus ou moins immédiate, selon les situations, d'un bibliothécaire professionnel, dans tous les types de bibliothèques: d'enseignement, publiques ou spécialisées, et même éventuellement à assumer la direction d'un personnel subalterne ou la responsabilité de services publics ou techniques.

Outre les matières spécifiquement professionnelles (aperçu des ressources de la bibliothèque, référence, catalogage et classification, équipement et techniques de bibliothéconomie, manutention du matériel spécial, publications gouvernementales - 8 heures/semaine), l'enseignement comporte des sujets plus techniques (dactylographie, publicité et expositions en bibliothèque, introduction aux appareils audio-visuels, informatique, administration - 7 heures/semaine), ainsi que des disciplines (en partie à option) choisies en relation étroite avec les coordonnateurs. Celles-ci se veulent une ouverture sur les principales disciplines du savoir. A cause de leur aptitude traditionnellement reconnue à imprimer un fonds de culture générale, elles créent une garantie de plus grande facilité pour le catalogage, la classification et la référence (langue maternelle, langue seconde obligatoire, sociologie, littérature, etc. - 12 heures/semaine). A cela, il faut ajouter des stages pratiques ( 2 heures/semaine). On en arrive ainsi à la proportion idéale souhaitée par I'A.L.A. et la C.L.A., soit approximativement $50 \%$ de cours de culture générale, $25 \%$ de bibliothéconomie, $25 \%$ de sujets connexes. ${ }^{1}$

II s'agit là d'un tableau d'ensemble dont les caractéristiques n'appartiennent pas, bien

1. John M. Marshall and June E. Munro, "Summary of a survey of library technician training programs in Alberta, British Columbia, Manitoba, Ontario, Quebec and Saskatchewan," Feliciter, vol. 18, no. 7 (1972), 5-6. 
sûr, exclusivement à Algonquin, et que l'on retrouve, à des degrés divers, dans les autres établissements du même genre, au Québec et en Ontario, offrant l'option en question. Aussi, ce que le présent article voudrait surtout faire ressortir, ce sont, outre le bilinguisme institutionnel, les efforts déployés par Algonquin pour instaurer des éléments de climat et d'orientation communautaires à l'intérieur du programme de bibliotechnique, à savoir l'attention suivie portée à chaque étudiant et l'utilisation des ressources abondantes, tant au point de vue compétence professionnelle qu'au point de vue collections, que possède la région d'Ottawa. De là un triple réseau d'activités qui se recoupent inévitablement les unes les autres tout en ayant leur configuration propre: la présence à l'étudiant, les stages d'apprentissage, les comités consultatifs.

\section{Les étudiants}

L'option "Bibliotechnique" compte actuellement, au secteur francophone, à temps plein, 18 étudiants en première année et 12 en deuxième; à temps partiel (éducation permanente), 20; du côté anglophone, 20 dans chacune des deux premières catégories et 100 dans la troisième. Soulignons en passant que le programme du soir, d'ailleurs équivalent en tous points à celui du jour, s'étend sur un minimum de 4 années et comporte une répartition plus concentrée des cours (130 crédits en tenant compte de l'expérience des élèves inscrits et de leur formation antérieure, étalés sur 15 semaines par semestre, soit hebdomadairement de 6 à 8 heures de cours.) Le décalage de 220 heures par rapport à l'horaire régulier se justifie par le fait que les étudiants ont plus d'expérience en bibliothèque et manifestent habituellement une motivation supérieure.

Le nombre d'inscriptions dans les diverses sections est évidemment plus élevé du côté anglophone, ce qui reflète la proportion de chaque groupe linguistique dans la population. II s'exerce cependant dans l'un et l'autre cas un processus très sévère de sélection à l'entrée, processus régi par un comité de trois professeurs, de telle sorte que le Collège possède en fait un contrôle total sur toutes les admissions. En outre, le mécanisme d'élimination se poursuit en cours d'études. Ceci est rendu possible non seulement grâce aux indications fournies par les résultats scolaires qui reflètent succès et insuccès, mais d'abord et surtout par l'examen presque quotidien des progrès de chaque sujet, spécialement à l'occasion des projets de recherche et des interviews.
Le processus de sélection est précédé, au cours de l'année, d'une série de visites dans les écoles secondaires de la région desservie afin de promouvoir la carrière "Bibliotechnique" et d'y intéresser les candidats possibles. Le coordonnateur s'y fait habituellement accompagner d'un étudiant de première ou de deuxième année. C'est là une innovation louable, parce que les étudiants du secondaire peuvent discuter avec quelqu'un de leur niveau et s'enquérir plus immédiatement de la vie du milieu telle que perçue par les intéressés eux-mêmes. Détail non négligeable: si la plupart des candidats possèdent une $12 \mathrm{e}$ année ou l'équivalent, beaucoup ont complété une $13 \mathrm{e}$ année, quelques-uns sont même détenteurs d'un B.A.

Une fois accepté et inscrit, l'étudiant jouit d'une attention soutenue de la part des coordonnateurs. Plusieurs moyens sont utilisés à cette fin: remise individuelle des travaux, discussion de la note finale du semestre avec l'étudiant concerné, rencontre des coordonnateurs avec les autres professeurs une ou deux fois par semestre afin de discuter spécifiquement du progrès ou des difficultés de chaque individu, rapports fournis par les diverses bibliothèques à l'occasion des stages pratiques. Il est possible par là de déceler non seulement si l'élève assimile bien la matière, mais encore s'il possède vraiment les qualités humaines et intellectuelles nécessaires à une profession bien intégrée à sa personnalité.

Une autre caractéristique du programme est l'étroite collaboration qui existe entre la bibliothèque du Collège et le programme de bibliotechnique. II ne s'agit en l'occurence, ni d'un moyen facile d'obtenir une maind'oeuvre à bon-marché, ni d'une formule teintée d'exclusivisme, au sens où les élèves seraient limités à l'utilisation de ces seules ressources. Bien au contraire. Mais il semble logique, étant donné la proximité des lieux, que plusieurs travaux scolaires soient poursuivis dans la bibliothèque, et que l'on s'en serve au maximum pour enseigner où et comment trouver l'information. La bibliothèque est ouverte aux étudiants en tout temps - ils y ont d'ailleurs un local permanent leur servant de laboratoire - et son utilisation permet d'éviter le double emploi en ce qui concerne en particulier les outils bibliographiques. Dans la mesure du possible, quelques projets seront orientés vers les besoins de la bibliothèque, de façon à leur conférer une dimension plus immédiatement communautaire: tout en contribuant à la formation, produire des éléments de travail qui peuvent demeurer sur place et servir à l'usage des autres étudiants. Ainsi des bibliographies, des expositions, des catalogues de périodiques, des dossiers documentaires, etc. 


\section{Les stages}

Les stages pratiques jouent un rôle de premier plan dans la formation du bibliotechnicien telle qu'envisagée par le Collége Algonquin. Outre les visites occasionnelles aux bibliothèques et les courts stages liés à des projets particuliers (ainsi les étudiants utilisent-ils la bibliothèque de l'Université d'Ottawa pour leurs recherches en référence II: ouvrages spécialisés) une résidence obligatoire annuelle de deux semaines dans une bibliothèque fait partie intégrante du programme. De plus, jusqu'ici, tous les étudiants, grâce aux efforts conjugués des coordonnateurs et du comité consultatif, y ont effectué un travail rémunéré durant les vacances d'été pour des périodes allant parfois jusqu'à quatre mois. Ce qui conduit souvent à un emploi permanent, une fois le diplôme obtenu. Ces expériences sont contrôlées, comme il a été dit plus haut, par le moyen de rapports fournis par les bibliothèques participantes, ce qui permet d'établir les points de force et de faiblesse des stagiaires.

De cette façon, la formation n'est pas restreinte aux catégories théoriques, et les étudiants peuvent accéder au marché du travail avec une connaissance concrète du genre de tâches qu'ils auront à accomplir, dussent-ils, selon les situations ultérieures, acquérir une plus grande spécialisation. D'autre part, la formule permet l'évaluation progressive en cours d'études des potentialités sociales, de la maturité et de la facilité d'adaptation des étudiants.

\section{Les comités}

Toutes ces initiatives aux divers paliers de la théorie et de la pratique se voient fortement appuyées, orientées, et sont parfois même engagées, par les comités consultatifs. Outre le comité provincial (Provincial Consultative Committee on Library Technician Training), il existe aussi à Algonquin un comité consultatif local. Le premier, qui représente un large éventail d'organismes professionnels, éducatifs et gouvernementaux, joue plutôt un rôle de supervision générale et de liaison.

II définit le rôle du technicien par rapport aux autres catégories de personnel de bibliothèque, fixe les normes d'établissement des écoles (endroit où il existe plusieurs types de bibliothèques, marché favorable), réglemente les conditions d'admission, détermine les critères d'enseignement sur la base des recommandations de la CLA (par exemple: "The difficulty is that this program still has only two full-time instructors, when it is actually two programs in one, and according to minimum standards should have four full-time instructors".) ${ }^{2}$ établit les qualifications du corps enseignant (au moins 2 ans d'expérience dans une bibliothèque -5 selon les nouvelles normes - inéligibilité d'une personne occupant un poste de direction dans la bibliothèque du Collège), contrôle le contenu des cours, organise des conférences et des séminaires pertinents. II veille en outre au caractère adéquat des ressources d'enseignement: bibliothèque d'au moins 25,000 volumes (40,000 selon les nouvelles normes) avec un fonds de 300 périodiques (600 selon les nouvelles normes) espaces suffisants pour les laboratoires, collection d'outils de référence autres que ceux de la bibliothèque pour l'utilisation en classe (laboratoires)

Le second comité, de composition locale, est un organisme obligatoire selon les normes précitées. Se réunissant un minimum de quatre fois par année, il comprend $15 \mathrm{mem}$ bres représentant les différents types de bibliothèques de la région, donc bien au fait de leurs opérations, de leurs orientations et de leurs exigences. Ce comité se divise luimême en deux sous-comités, qui se rencontrent selon les besoins: l'un s'occupe de l'emploi, faisant périodiquement enquête sur les possibilités du marché du travail dans la région; l'autre des programmes, suggérant les modifications souhaitables, surtout en ce qui concerne les cours à option et les matières liées à la bibliotechnique. Ainsi, l'an dernier, suggérait-il l'établissement de cours d'expositions dans une bibliothèque et de procédures d'affaires.

L'action du comité local en particulier s'avère, dans la pratique, extrêmement fructueuse. Ses membres, en plus de jouer un rôle de liaison entre le Collège et les employeurs éventuels, en s'occupant dans leur secteur respectif de s'informer des possibilités d'emploi, aident à planifier les stages d'apprentissage, veillent à l'application immédiate des normes ontariennes, établissent, à partir des conditions du marché le nombre optimal des admissions. Ce travail de coordination porte ses fruits. A I'heure actuelle, tous les diplômés d'Algonquin ont un travail permanent. Des 12 diplômés francophones, 2 oeuvrent dans les bibliothèques publiques, 1 dans une bibliothèque universitaire, 3 dans les écoles secondaires, 1 à la Bibliothèque nationale du Canada, 5 dans des bibliothèques spécialisées. Du côté anglophone, dans le même ordre, les chiffres sont de 2,3, 6,0,8. Ce sont là des résultats tangibles qui reflètent

\footnotetext{
2. John M. Marshall and June E. Munro, "Summary of ...", 14.
} 
à tout le moins une certaine efficacité du régime pédagogique et des structures de relations publiques.

\section{Conclusion}

II ne fait pas de doute - et on l'a souvent souligné - que les bibliotechniciens, à cause de la formation poussée qu'ils reçoivent un peu partout au Canada ${ }^{3}$, pourront de plus en plus accomplir des tâches actuellement remplies par les professionnels, libérant ceux-ci pour des fonctions plus spécialisées (bibliographie, référence, administration, etc.). II est à souhaiter que les bibliothécaires voient l'arrivée des bibliotechniciens non comme une menace à leur statut, mais plutôt comme une stimulation à se perfectionner davantage, afin d'exceller là où peut-être auparavant ils se contentaient d'une honnête compétence. Voir dans un esprit d'antagonisme et en termes de concurrence le nouveau rapport de forces qui se dessine serait non seulement malsain pour le climat général de travail, mais démontrerait on ne peut mieux ce que plusieurs déjà nous reprochent, à savoir un statisme rebelle aux défis allié à un chauvinisme professionnel outré qui, pour trop embrasser, mal étreint.

3. Voir le tableau de Feliciter, art. cit., et Ministère de l'Education du Québec. Cahiers de l'enseignement collégial 1971-72.
A cet égard, l'envahissement des domaines techniques par les bibliotechniciens (catalogage, classification, etc.) nous forcera peut-être à réévaluer notre philosophie, à nous resituer par rapport aux occupations classiques, et à nous faire comprendre que l'exercise de la bibliothéconomie doit être une profession où le véritable humanisme, la culture et même l'érudition peuvent jouer un rôle au moins aussi considérable que l'application minutieuse et parfois bornée de règles, de procédures et de prescriptions, catalographiques ou autres. Ce que certains esprits chagrins entrevoient comme une nouvelle invasion des Barbares, et que l'on décrie, ne pourrait-il pas devenir simplement, pour nous, l'occasion d'une renaissance de l'esprit d'invention?•
- Le premier Répertoire de l'édition au Québec réalisé à l'aide d'un ordinateur

- Près de 10000 titres disponibles en librairie au 30 juin 1972

- INDEX DES AUTEURS

- INDEX DES TITRES

- INDEX DES ÉDITEURS

- INDEX DES COLLECTIONS

- LISTE DES LIBRAIRIES AGREEEES

- Index alphabétique et numérique ISBN

- Liste des organismes professionnels francophones du livre dans le monde

- Liste des membres des associations professionnelles québécoises du livre

- Liste des revues et périodiques bibliographiques et littéraires du Canada

- Liste des publications sur le livre dans le monde

UN VOLUME CARTONNÉ $81 / 2^{\prime \prime} \times 12^{\prime \prime}, 366$ pages: $\$ 15$.

\section{ENFIN DISPONIBLE RÉPERTOIRE DE L'ÉDITION AU QUÉBEC 1972}

\section{PUBLIÉ PAR L'ASSOCIATION DES EDITEURS CANADIENS ET \\ LA SOCIÉTÉ DES ÉDITEURS DE MANUELS SCOLAIRES DU QUÉBEC}

avec le concours du ministère des Affaires culturelles du Québec, de la Bibliothèque Nationale du Québec et la collaboration du Conseil Supérieur du Livre. disponibles en librairie au 30 juin 1972 , comprenant les titres déposés à la Bibliothèque Nationale du Québec à la même date.

Distributeur exclusif:

LES PRESSES DE L'UNIVERSITE

C.P. 2447, QUÉBEC 2, CANADA LAVAL 